International Journal of Engineering \& Technology, $7(2.6)(2018) 290-292$
International Journal of Engineering \& Technology
SPC
Website: www.sciencepubco.com/index.php/IJET
Research Paper

\title{
Semantic Ranking based on Keywords Extractions in the web
}

\author{
A Sangeetha ${ }^{1}$, C. Nalini ${ }^{2}$ \\ ${ }^{1}$ Research scholar, Department of Computer Science \& Engineering, BIHER, BIST, Bharath University, Chennai, India \\ ${ }^{2}$ Professor, Department of Computer Science \& Engineering, BIHER, BIST, Bharath University, Chennai, India \\ *Corresponding author E-mail: sangeethagsam@gmail.com
}

\begin{abstract}
The collection of terminologies from text is animportant for several issues, i.e., the future extraction and also summarization of keyword in semantic network. Semantic Ranking is typically performed using statistical data from text. Our proposed strategy develops a semantic chart utilizing understood connections, which depend on semantic relatedness between content hubs and therefore positions hubsutilizing Ranking calculations. This research work focuses only Semantic Rank for keyword mining from text. This method for keywords extraction shows that Semantic Rank makesconstructivelyin the data set.
\end{abstract}

Keywords: Sysnet, Semantic, Ontology, Hypernym, Hyponym.

\section{Terms and Methods}

In this section describes the terms and methods of this research work. A hyponym is a word else expression whose semantic field is incorporated inside of some other word, its hypernym or hyperonym.In less difficult terminologies, a hyponym imparts a kind of relationship to its hypernym. Such as, pigeon,seagull, crowand hawk are generally hyponyms of flying creature (their hypernym); these are all hyponym of creature.

Before constructing a word cloud, the input is preprocessedby using the following steps:

Term Extraction:The input is first split into sentences, and then tokenized all keywords into a group of words. In frequent stopwords such as "a", "the", "is" are detached from the group. The most commonly recognized variety of the word is exploited as a part of the last word cloud.

Ranking: In the subsequent stage we rank the words arranged by relative significance. We utilize three distinctive positioning capacities, contingent upon word utilization in the info content. Each positioning capacity orders words by their relegated weight (rank).
Similarity Computation: Given the positioned rundown of words, we ascertain a network of pairwise similitudes with the goal that related words get high comparability esteems.

The system creates word clouds using several sources of textual data.

The simplest source is a text document entered by a user. Users specify the URL of a webpage or the connection to a PDF document. In this case, a word is build derived from the extracted text. Another preference is to specify the connection to a Facebook. For the scenario, the system parses all comments for the video and produces a "comment cloud".

The fundamental segment of this engineering is the User, which gets all these client collaborations and produces another arrangement of labels which will be utilized by the Web Interface as look terms for new pictures. To create these new labels, the User Profiler utilizes two free modules: WordNet and LAr.

LAr is a formal adaptive logic for abduction. This module has just been actualized in Ruby. WordNet is an expansive browseable lexical database, accessible in a few dialects, which bunches synsets communicating an unmistakable idea. We utilize WordNet as a help instrument for the recovery of semantic learning related to labels.

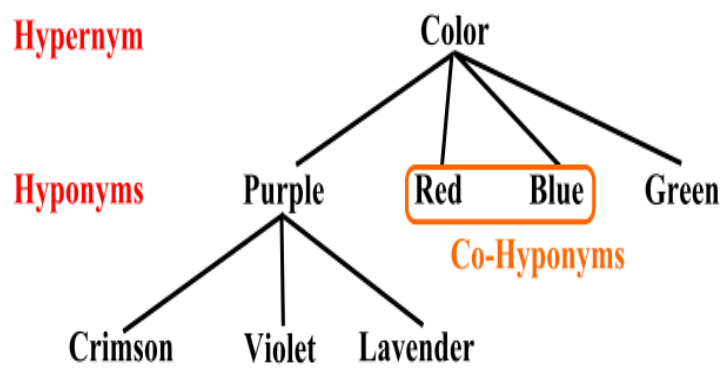

Fig. 2: Representation of Hypernyms \& Hyponyms. 


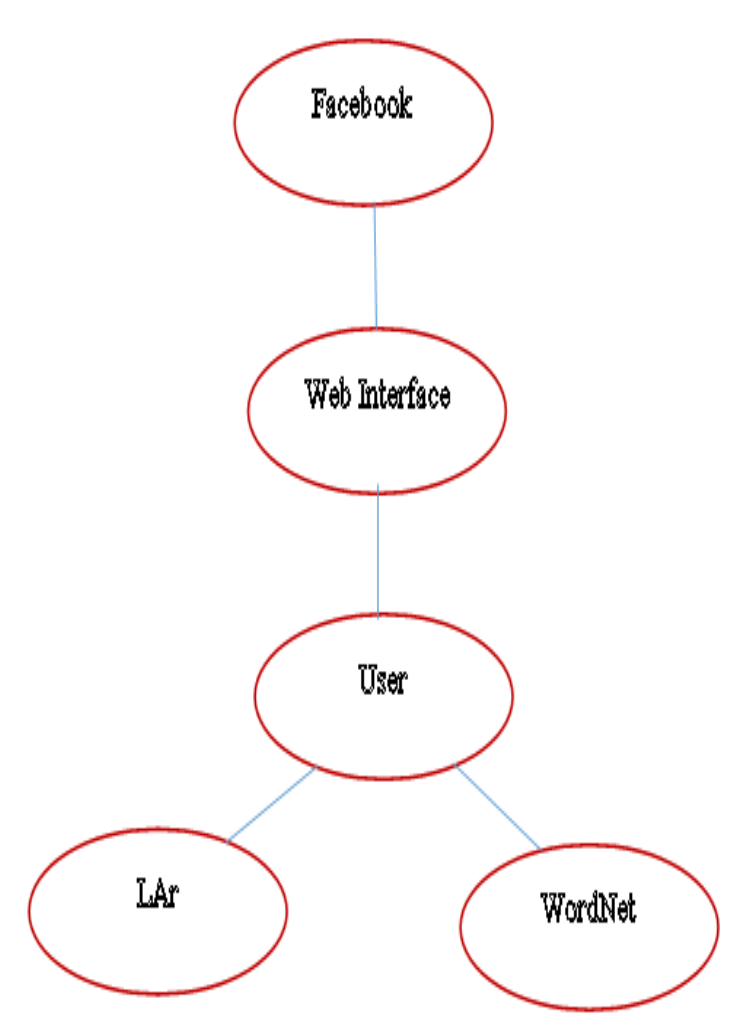

Fig. 3: Flow of Ontology segments

\section{Experimental Setup}

Here clearly demonstrates about the experimental set up of the semantic ranking depended on the Programming Language ontologies contain a few models that specialists use to break down the conduct of Programming productivity and transportability. These ontologies are made to empower the sharing and reuse of programming dialect models among philosophy instruments and their clients. Among the different programming ontologies, the Top down and Bottom up approach ontologies are watched.

Here, WordNet based semantic selector that assembles with a semantic weighted distributed likelihood. We have talked about the approach by disclosing a gathering of semantic measures, and also others entirely control to the structure investigation. A while later, we chose an entropy-based estimation in view of its included mix of chart structure and likelihood dissemination examination over the diagram.

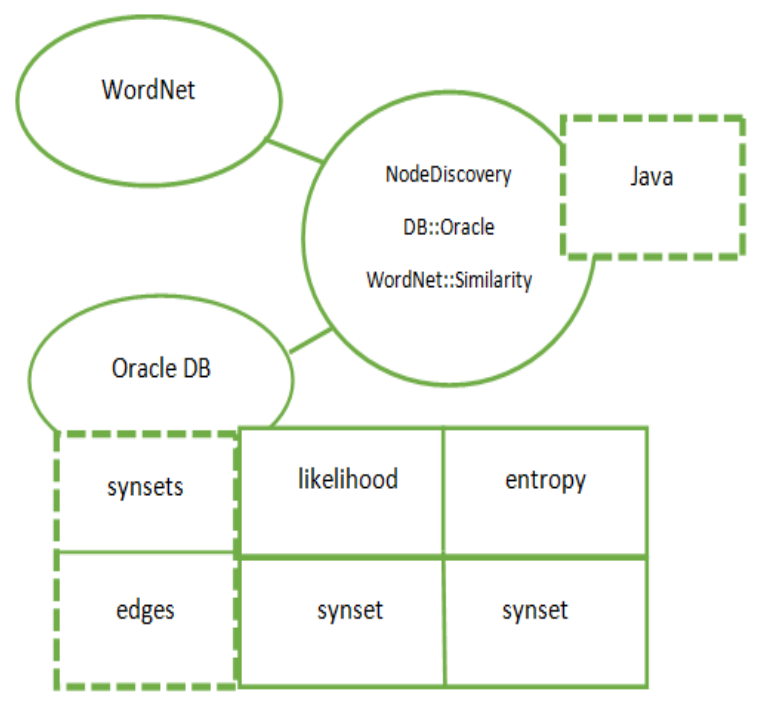

Fig. 4: Word sense ranking mechanism of Ontology
At the point when the Programming Languages ontologies were outlined, the designers had three sorts of uses as a top priority. To start with, these ontologies ought to give a machine and humanreadable notation for speaking to the models and area speculations found in the languages literature. Second, they ought to give a formal detail of a shared conceptualization and a vocabulary for a group of interoperating software agents in scripting languages areas. Furthermore, third, they should put the base for other formalization endeavors including more thorough ontologies for area particular dialects.
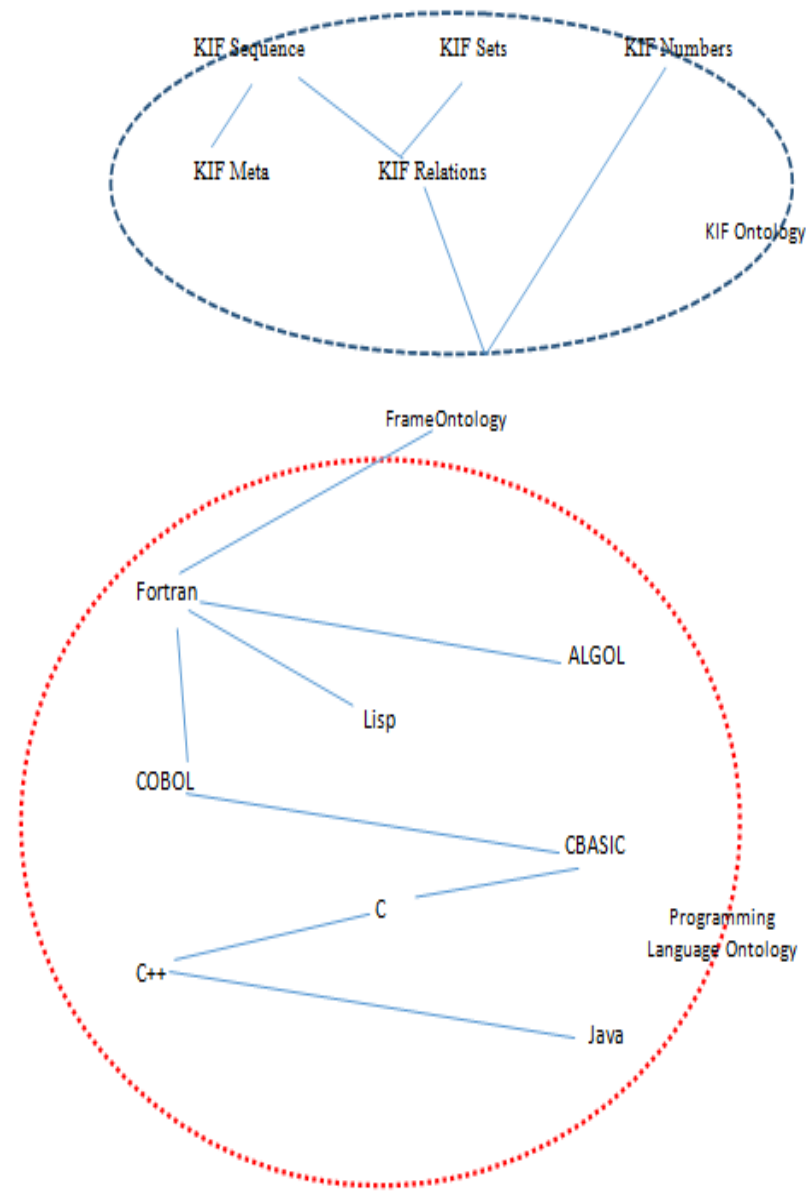

Fig. 5: Structure of the Programming Language ontologies

\section{Results and Analysis}

Here depicted the table clearly demonstrates the java is having highest semantic ranking compare than others. FORTRAN is having lowest entropy compare than some alternate programming technologies ontology. C++, C, CBASIC, COBOL, Lisp, ALGOL are having moderate entropy ranking in the semantic ranking. We at that point utilized a nearby semantic assess to compute this likelihood, empowering thus the entropy figuring.

Table 1: Representation of Synset entropies

\begin{tabular}{|l|l|c|}
\hline \multicolumn{1}{|c|}{ synset } & \multicolumn{1}{c|}{ likelihood } & entropy \\
\hline java \#n\#1 & 0.00335609162096442 & 0.915167893638109 \\
\hline C++\#n\#1 & 0.00266239416637492 & 0.914798934400538 \\
\hline C\#n\#1 & 0.00238034135516821 & 0.914588208088081 \\
\hline $\begin{array}{l}\text { CBASIC\# } \\
n \# 1\end{array}$ & 0.0022103296355282 & 0.913835110905649 \\
\hline $\begin{array}{l}\text { COBOL\# } \\
n \# 1\end{array}$ & 0.00049930187054021 & 0.913797186379224 \\
\hline Lisp\#n\#3 & 0.00058396100572906 & 0.913357856810317 \\
\hline $\begin{array}{l}\text { AL- } \\
\text { GOL\#n\#1 }\end{array}$ & 0.0015588248704844 & 0.912980543614797 \\
\hline $\begin{array}{l}\text { FORTRA } \\
\text { N\#n\#2 }\end{array}$ & 0.00127960854260848 & 0.912779233401893 \\
\hline
\end{tabular}




\section{Conclusions}

We portrayed the method used to gauge the relative importance of every hub by the impact of its evacuation over the worldwide diagram entropy. This measurement is uncommonly suited to finish the determination of abductive inferences over WordNet in an ideation procedure on the grounds that the connected versatile snatching rationale, despite the fact that has recursive intelligibility location, this research work recommended the java programming language is having highest semantic ranking compare than others.

This research work proves that java programming language had highest semantic ranking, a further extension of the work is required to prove some modern procedural language ranking compare with java programming language.

\section{References}

[1] B. Prasanthi, Suresh Pabboju, D. Vasumathi, "Query Adaptive Hash Based Image Retrieval In Intent Image Search", Journal of Theoretical and Applied Information Technology, vol. 93, no. 2, November 2016.

[2] Rasika V. Khandre, Neeta A. Deshpande, Rasika V. Khandre et al., "A Review on Web Image Retrieval Techniques", (IJCSIT) International Journal of Computer Science and Information Technologies, vol. 6, no. 2, pp. 1209-1212, 2015.

[3] A Ramachandran, M Sai Kumar, C. Nalini, "Learning Image ReRank: Query-Dependent Image Re-Ranking Using Semantic Signature", International Journal of Innovative Research in Science Engineering and Technology (An ISO 3297: 2007 Certified Organization), vol. 4, no. 3, March 2015.

[4] Vaamonde et al., "What Can Pictures Tell Us About Web Pages? Improving Document Search Using Images", IEEE Trans. on Pattern Analysis and Machine Intelligence, vol. 37, no. 2015, pp. 1274-1285.

[5] Distinto I., d'Aquin M., Motta E.LOTED2: An ontology of European public procurement notices Semant. Web, 7 (3) (2016) pp. 267-293

[6] M. Lee, N. Matentzoglu, B. Parsia, U. Sattler, A multi-reasoner, justification-based approach to reasoner correctness, in: The Semantic Web - ISWC 2015 - 14th International Semantic Web Conference, Bethlehem, PA, USA, October 11-15, 2015, Proceedings, Part II, 2015, pp. 393-408.

[7] B. Parsia, N. Matentzoglu, R.S. Gonçalves, B. Glimm, A. Steigmiller, The owl reasoner evaluation (ORE) 2015 competition report, in: Proceedings of the 11th International Workshop on Scalable Semantic Web Knowledge Base Systems (SSWS2015), Bethlehem, Pennsylvania, USA, October 11, 2015.

[8] Slack E. How much local fiscal autonomy do cities have? A comparison of eight cities around the world IMFG Perspectives, 19 (2017)

[9] Fox M.S. The role of ontologies in publishing and analyzing city indicators Computers, Environment and Urban Systems, 54 (2015), pp. 266-279

[10] Wang Y., Fox M.S. Consistency analysis of city indicator data Planning Support Systems for Smarter Urban Futures, Springer (2017)

[11] Pereira R.L., Sousa P.C., Barata R., Oliveira A., Monsieur G. CitySDK tourism API -building value around open data Journal of Internet Services and Applications, 6 (24) (2015)

[12] M.S. Fox, C. Pettit, On the Completeness of Open City Data for Measuring City Indicators, Proceedings of the IEEE Conference on Smart Cities, Guadalajara Mexico, 2015.

[13] S. Bischof, C. Martin, A. Polleres, P. Schneider, Collecting, integrating, enriching and republishing open city data as linked data, Proceedings of the14th International Semantic Web Conference, Bethlehem, PA, USA, 2015, pp. 57-75.

[14] S. Bischof, B. Kampgen, A. Harth, A. Polleres, P. Schneider Open City Data Pipeline, Working Papers on Information Systems, Information Business and Operations, No. 01/2017, Department of Information Systems and Operations, Vienna University of Economics and Business, 2017.

[15] Otero-Cerdeira L., Rodriquez-Martinez F.r., Gomex-Rodriguez A.Ontology matching: A literature review Expert Syst. Appl., 42 (2) (2015), pp. 949-971. 